\title{
Incidencia del medio ambiente académico en la formación biomédica
}

\section{Impact of the academic environment in biomedical training}

\author{
Karen Galaviz Loya ${ }^{1}$, Brenda Amador Negrete ${ }^{2}$ y Haydeé Parra Acosta ${ }^{3^{*}}$ \\ ${ }^{1}$ Facultad de Medicina y Ciencias Biomédicas. Universidad Autónoma de Chihuahua. (México). \\ galavizkaren14@gmail.com https:/ / orcid.org/0000-0003-2535-7412 \\ ${ }^{2}$ Facultad de Medicina y Ciencias Biomédicas. Universidad Autónoma de Chihuahua. (México). \\ brendaamador034@gmail.com https://orcid.org/0000-0003-0284-0356. \\ ${ }^{3}$ Departamento de Investigación Educativa. Facultad de Medicina y Ciencias Biomédicas. Universidad \\ Autónoma de Chihuahua (México) hparra05@hotmail.com. https://orcid.org/0000-0003-1720-7182 \\ *Correspondencia: hparra05@hotmail.com. Circuito Universitario. Campus II. C.P. 31125.
}

Recibido: 10/11/2020; Aceptado: 25/1/2021; Publicado: 1/2/2021

Resumen: Entre los aspectos que conforman el ambiente académico, se encuentran las habilidades y actitudes de los docentes, la comunicación entre docentes y estudiantes, la infraestructura, duración de clases y carga académica, los sistemas de apoyo y la seguridad personal. El presente artículo expone los resultados de una investigación que intenta responder la siguiente interrogante: ¿En qué medida los aspectos del medio ambiente académico inciden en la formación biomédica de los estudiantes? Los objetivos de investigación son: 1) Identificar los elementos del medio ambiente mejor valorados por parte de estudiantes y docentes, 2) Determinar si existen diferencias significativas en las percepciones que tienen los docentes y estudiantes respecto al ambiente académico; asimismo, 3) Identificar los aspectos del ambiente académico que contribuyen a la formación biomédica. Es un estudio transversal donde se aplicaron cuestionarios previamente validados a 185 estudiantes y 50 docentes. En los resultados sobresale, que lo mejor evaluado del medio ambiente es la actitud ética del docente y habilidades respecto a propiciar el estudio auto dirigido y/o autorregulado, el pensamiento complejo y crítico, así como su experiencia docente. Se observaron diferencias significativas entre los docentes y estudiantes respecto a las variables que integran el ambiente académico. Asimismo, resaltamos que un ambiente académico que garantiza la formación integral como médico se correlaciona significativamente con que los estudiantes se formen en valores, ética, trabajen de forma colaborativa y posean los conocimiento necesarios para el manejo integral del paciente.

Palabras clave: Educación Médica; Ambiente Académico; Estudiantes; Docentes.

Abstract: Among the aspects that integrate the academic environment are the skills and attitudes of teachers, communication between teachers and students, infrastructure, duration of classes and academic burden, support systems and personal safety. This article sets out the results of research trying to respond the following research question: What extent do aspects of the academic environment affect students' biomedical training. The objectives are: 1) to identify the best-valued elements of the environment by students and teachers, 2) to determine whether there are significant differences in teachers' and students' perceptions of the academic environment, 3) to identify aspects of the academic environment that contribute to biomedical training. It is a cross-cutting study where previously validated questionnaires were applied to 185 students and 50 teachers. In the results, it stands out that the best evaluated of the environment is the ethical attitude of the teacher and skills regarding promoting self-directed and/or self-regulated study, complex and critical thinking, as well as his experience. Significant differences were observed between teachers and students, compared to the variables that make up the academic environment. It also emphasizes in this study, that an academic environment that guarantees comprehensive training as a physician, correlates significantly with students training in values, ethics, working collaboratively and possessing the necessary knowledge for the integral management of the patient.

Keywords: Medical Education; Academic Environment; Students; Teachers. 


\section{Introducción}

La formación es un proceso que se construye en interrelación permanente entre el individuo y el medio (1). Debido a esto, el concepto de medio ambiente académico (MAA) es un tema de análisis y discusión relevante ya que se encuentra asociado al éxito y satisfacción percibida por los estudiantes. El MAA es un constructo amplio que hace referencia a las condiciones materiales e intangibles de una institución, incluyendo relaciones entre las personas y características de estas interacciones (2). Su definición involucra las impresiones, creencias y expectativas que los miembros de una comunidad educativa tienen de su propia escuela, las conductas asociadas y los símbolos y estamentos que representan dichas conductas. Entre los aspectos que constituyen el ambiente académico se encuentran las habilidades y actitudes de los docentes y estudiantes, la comunicación entre docentes y estudiantes, la infraestructura de la facultad, la duración de las clases y la carga académica, los sistemas de apoyo, las tecnologías de la información y comunicación (TICs) y la seguridad personal (3-4). Todos son importantes, ya que de ellos depende la calidad del aprendizaje, el respeto por la enseñanza y la confianza en los profesores (3). En este sentido, el ambiente académico se ha identificado como uno de los aspectos que favorece la implementación de un plan de estudio eficaz, por lo que los docentes médicos han reconocido los efectos que tiene sobre la construcción a nivel académico y clínico, así como en el desarrollo de competencias de los estudiantes de medicina (4).

Los procesos formativos por competencias, en el contexto actual, se realizan desde una perspectiva integral del contexto y con un enfoque complejo, que afirma la igualdad de los seres humanos y fortalece los valores en las interacciones que se establecen con los otros y con la comunidad. En este sentido, la formación biomédica reconoce la diversidad y la inclusión de lo diferente como una oportunidad de crecimiento personal y comunitario y además promueve un compromiso con quienes más lo necesitan (5). Por lo que es práctico, dinámico, multifactorial y transdisciplinar, orientado a desarrollar el máximo potencial de los estudiantes.

Los problemas de salud pública a los que nos enfrentamos actualmente, requieren médicos competentes que promuevan la salud de forma humanista. Médicos que contribuyan a mejorar calidad de vida de las personas con una visión prospectiva humanista (6) para que brinden una atención médica de alta calidad y enfrenten con responsabilidad los desafíos actuales de su profesión. Por lo que una de las tareas del tutor-docente es la de formar al médico en la bioética. Responsabilidad que incluye requerimientos de orden ético en los docentes y estudiantes (integridad, aptitud, conocimientos, experiencia, respeto, sinceridad, justicia, humildad, prudencia y ejemplaridad) en el mayor grado posible (7).

Los modelos pedagógicos buscan fomentar en los estudiantes una actitud positiva hacia el aprendizaje, considerando su papel protagonista en la construcción del conocimiento, y los integra en un ambiente de participación y cooperación para que contribuyan a la solución de problemas de salud, mostrando apertura para aprender de los demás y para comunicarse con los miembros de un grupo o sus pares (8). De acuerdo con ello, es necesario generar ambientes de aprendizaje que promuevan la interacción entre los docentes y estudiantes en clima de confianza y de apertura, que garanticen una educación con calidad y equidad (7). Es así que el interés por analizar los aspectos relacionados con el MAA y valorar su incidencia en la formación médica, se ha incrementado a fin de establecer estrategias para la implementación de modificaciones curriculares que permitan innovar la formación médica en las instituciones educativas (9) para enfrentar los retos y desafíos actuales. Para ello, se han diseñado diferentes instrumentos como el Dundee 
Ready Educational Environment Measure (DREEM), Postgraduate Hospital Educational Environment Measure (PHEEM) y Clinical Learning Environment Scale (CLE). Sin embargo, se carece de información actual que muestre los aspectos del medio ambiente educativo que favorecen el proceso formativo del médico desde una perspectiva integral y humanista. De esta manera, en el presente artículo, se presentan los resultados de una investigación que intenta responder a la siguiente interrogante: ¿En qué medida los aspectos del medio ambiente académico inciden en la formación biomédica de los estudiantes desde una perspectiva integral y humanista? Para ello, se planteó la siguiente hipótesis: existe correlación significativa entre un ambiente académico y la formación biomédica cuando este garantiza la formación integral, una calidad con inclusión y contribuye a los estudiantes aprendan a aprender, realicen investigación médica, se formen en valores y ética, trabajen de forma colaborativa y posean los conocimientos necesarios para el manejo integral del paciente.

Los objetivos que se plantearon para responder a esta interrogante son: 1) Identificar los elementos del medio ambiente mejor valorados por parte de estudiantes y docentes. 2) Determinar si existen diferencias significativas en las percepciones que tienen los docentes y estudiantes respecto al ambiente académico. 3) Identificar los aspectos del ambiente académico que contribuyen más a la formación biomédica, integral y humanista.

\section{Métodos}

El diseño del estudio es transversal y se realizó mediante la aplicación de cuatro cuestionarios que fueron dirigidos a estudiantes de ciencias básicas y estudiantes de ciencias clínicas, docentes de ciencias básicas y ciencias clínicas. Los instrumentos se diseñaron considerando algunas de las variables del cuestionario del DREEM junto con otros cuestionarios y la revisión sistemática de varios artículos que explican las variables del estudio. Los cuestionarios integran tres variables demográficas y unas 90 variables simples organizadas en dos dimensiones: Medio Ambiente y Formación Biomédica, que se validaron mediante la aplicación de cuatro grupos piloto conformados por 40 estudiantes de básicas, 25 estudiantes de clínicas, 26 docentes de básicas y 20 docentes de clínicas, para analizar la confiabilidad y consistencia interna a través del estadístico alfa de Cronbach.

La población de estudio son 426 docentes y 1607 estudiantes de ciencias básicas y clínicas de la carrera de Médico Cirujano y Partero de la Facultad de Medicina y Ciencias Biomédicas de la Universidad Autónoma de Chihuahua. El tamaño de la muestra se estimó con la fórmula de William G. Cochran (10), obteniéndose una muestra corregida de 236 personas, Con un error muestral de $0.06 \%$. Al estratificar la muestra, esta quedó conformada por 50 docentes y 186 estudiantes. La muestra final quedó con 190 estudiantes y 71 docentes de ciencias básicas y clínicas, obteniéndose un total de 261 respuestas como se muestra en la tabla 1.

Tabla 1. Respuestas obtenidas por grupo poblacional

\begin{tabular}{|c|c|}
\hline Población & No. De respuestas \\
\hline Estudiantes de Ciencias Básicas & 163 \\
\hline Estudiantes de Ciencias Clínicas & 27 \\
\hline Docentes de Ciencias Básicas & 32 \\
\hline Docentes de Ciencias Clínicas & 39 \\
\hline Total & 261 \\
\hline
\end{tabular}


Los criterios de inclusión fueron ser docente o estudiante de cualquier semestre de ciencias básicas o clínicas de la Facultad de Medicina y Ciencias Biomédicas de la Universidad Autónoma de Chihuahua. Las variables de estudio son el medio ambiente académico como variable independiente y la formación biomédica como variable dependiente (tabla 2).

Tabla 2. Definición de variables

\begin{tabular}{|c|c|c|c|}
\hline Variable & Concepto & Indicadores & Escala de medición \\
\hline $\begin{array}{l}\text { Variable } \\
\text { independiente: }\end{array}$ & $\begin{array}{l}\text { Medio ambiente } \\
\text { académico }\end{array}$ & $\begin{array}{l}\text { Actitudes de docentes y } \\
\text { estudiantes, habilidades de } \\
\text { comunicación, infraestructura de } \\
\text { la facultad, carga académica, } \\
\text { sistemas de apoyo, TICs y } \\
\text { seguridad personal }\end{array}$ & Escala Likert del 0 al 4: \\
\hline $\begin{array}{l}\text { Variable } \\
\text { dependiente: }\end{array}$ & $\begin{array}{l}\text { Formación } \\
\text { biomédica }\end{array}$ & $\begin{array}{l}\text { Incluye el manejo integral del } \\
\text { paciente, la revisión de casos } \\
\text { clínicos, el desarrollo del } \\
\text { pensamiento crítico y analítico, la } \\
\text { promoción de una educación } \\
\text { congruente con las necesidades } \\
\text { de salud, la formación del médico } \\
\text { humanista, el aprendizaje en la } \\
\text { interacción con los pacientes, } \\
\text { entre otros. }\end{array}$ & $\begin{array}{l}\text { 0, "Nunca/Deficiente/Nada" } \\
\text { 1, “Pocas veces/Regular/Poco" } \\
\text { 2, "Algunas veces/ } \\
\text { Bueno/Suficiente" } \\
\text { 3, “Frecuente/Muy } \\
\text { bueno/Bastante" } \\
\text { 4, "Siempre/ Excelente/ } \\
\text { Totalmente". }\end{array}$ \\
\hline
\end{tabular}

Con estas variables se diseñaron los cuatro cuestionarios digitales, buscando que los cuestionarios de docentes y estudiantes de ciencias básicas y los de ciencias clínicas llevaran el mismo número de variables o ítems y que fueran similares (tabla 3).

Tabla 3. Tipo de cuestionarios y número de variables.

\begin{tabular}{|c|c|c|c|}
\hline No. & Tipo de cuestionario & Dirigido a & $\begin{array}{c}\text { Total de } \\
\text { variables }\end{array}$ \\
\hline 1 & Estudiantes C. básicas & Estudiantes de $1^{\circ}$ a $5^{\circ}$ semestre & 90 \\
\hline 2 & Estudiantes C. clínicas & Estudiantes de $6^{\circ}$ a $11^{\circ}$ semestre & 97 \\
\hline 3 & Docentes C. básicas & Docentes de $1^{\circ}$ a $5^{\circ}$ semestre & 90 \\
\hline 4 & Docentes C. Clínicas & Docentes de $6^{\circ}$ a $11^{\circ}$ Semestre & 97 \\
\hline
\end{tabular}

El análisis de la información se realizó a través de la estadística descriptiva e inferencial de la forma siguiente. Se realizó un análisis exploratorio para valorar si el comportamiento de las variables era normal. Después, un análisis descriptivo de medias (univariante) para identificar variables con un valor superior o inferior de $\langle X-1 \sigma, X+1 \sigma\rangle$ y se determinó las variables sobresalientes de los límites de normalidad, denominándose atípico superior e inferior, que son las variables más y menos valoradas por los docentes y estudiantes. Se establecieron comparativos entre estudiantes y docentes de ciencias básicas y clínicas con relación a las variables que integran el medio ambiente y la formación 
biomédica a través de un anova simple con la prueba Tukey con un nivel de significación de $\mathrm{p}$ menor de 0.05 . También se realizaron correlaciones entre las variables que integran los aspectos relacionados con el ambiente educativo y la formación biomédica de los estudiantes, utilizando el criterio de Pearson de correlación media hacia alta (0.50-1.00). Para ello se realizaron análisis de regresión lineal para precisar los elementos que mejor predicen la formación biomédica.

Los análisis estadísticos se realizaron utilizando Statistical Package for the Social Siciences (SPSS 25) y Statistica 64 v.10. El proyecto de investigación fue aprobado por el comité de investigación CI-006-20, por lo que asegura la confidencialidad de la información y datos obtenidos en las encuestas según lo establecido en la Ley Federal de Protección de Datos Personales; en dichos instrumentos, se plasma la finalidad del proyecto $\mathrm{y}$, al ser de aplicación virtual, tanto estudiantes como docentes tuvieron la oportunidad de decidir participar o no.

\section{Resultados}

Los resultados de la validación del cuestionario a través del alpha de Cronbach que se utilizó para medir la consistencia interna de cada uno de los instrumentos mostraron un alto grado de confiabilidad (tabla 4), lo que permite generalizar los resultados a la población objetivo.

Tabla 4. Grado de Confiabilidad de los instrumentos.

\begin{tabular}{|c|c|}
\hline Instrumento & Valor de ac \\
\hline Estudiante Ciencias Básicas & 0.991 \\
\hline Estudiante Ciencias Clínicas & 0.994 \\
\hline Docente Ciencias Básicas & 0.966 \\
\hline Docente Ciencias Clínicas & 0.989 \\
\hline
\end{tabular}

\subsection{El medio ambiente educativo: análisis descriptivo.}

El análisis de medias reportó, de acuerdo con el valor atípico superior $X+1 \sigma=3.26$ que lo más valorado del medio ambiente por docentes y estudiantes fue: que los docentes demuestran una actitud ética, propician el estudio auto dirigido y autorregulado, propician el pensamiento complejo, el pensamiento crítico, demuestran experiencia en la materia que imparten $y$, que los servicios administrativos son eficientes. Por el contrario, lo menos valorado o bien, lo que sobresalió como atípico inferior $(X-1 \sigma=2.77)$ fue que el ambiente académico contribuye a realizar investigación médica, la facultad forma a los estudiantes para tener una vida saludable, con ejercicio y alimentación acorde con el plato del bien comer, la carga horaria permite que los alumnos estudien en casa y en la biblioteca y que los estudiantes y docentes conocen si la facultad cuenta con servicios de seguridad (tabla 5).

Tabla 5. Variables más y menos valoradas por docentes y estudiantes respecto al ambiente académico

\begin{tabular}{|l|c|l|c|}
\hline \multicolumn{1}{|c|}{ Más valorado } & Media/D.E. & \multicolumn{1}{c|}{ Menos valorado } & Media/D.E. \\
\hline $\begin{array}{l}\text { V.62 Los docentes } \\
\text { demuestran una actitud } \\
\text { ética }\end{array}$ & $3.27 \pm 0.96$ & $\begin{array}{l}\text { I.2. El ambiente } \\
\text { académico contribuye a } \\
\text { realizar investigación } \\
\text { médica }\end{array}$ & $2.55 \pm 1.14$ \\
\hline V.64. Los docentes & $3.38 \pm 0.91$ & II.23. La facultad forma & $2.29 \pm 1.33$ \\
\hline
\end{tabular}




\begin{tabular}{|l|l|l|l|}
\hline $\begin{array}{l}\text { propician el estudio } \\
\text { autodirigido y/o } \\
\text { autorregulado }\end{array}$ & $\begin{array}{l}\text { estudiantes para tener } \\
\text { una vida saludable, con } \\
\text { ejercicio y alimentación } \\
\text { acorde con el plato del } \\
\text { bien comer }\end{array}$ & \\
\hline $\begin{array}{l}\text { V.65. Los docentes } \\
\text { propician el } \\
\text { pensamiento complejo }\end{array}$ & $3.28 \pm 0.97$ & $\begin{array}{l}\text { II.28. La carga horaria } \\
\text { permite que los alumnos } \\
\text { estudien en casa y en la } \\
\text { biblioteca }\end{array}$ & $2.50 \pm 1.25$ \\
\hline $\begin{array}{l}\text { V.66. Los docentes } \\
\text { aplican estrategias para } \\
\text { propiciar el desarrollo } \\
\text { del pensamiento crítico }\end{array}$ & $3.34 \pm 0.91$ & $\begin{array}{l}\text { IV.53. Los estudiantes y } \\
\text { docentes conocen si la } \\
\text { facultad cuenta con } \\
\text { servicios de seguridad }\end{array}$ & $2.55 \pm 1.42$ \\
\hline $\begin{array}{l}\text { V.71. Los docentes } \\
\text { demuestran experiencia } \\
\text { profesional en la materia } \\
\text { que imparte }\end{array}$ & $3.42 \pm 0.94$ & $3.26 \pm 0.93$ & \\
\hline $\begin{array}{l}\text { VIII. 96. Los servicios } \\
\text { administrativos son } \\
\text { eficientes }\end{array}$ & & & \\
\hline
\end{tabular}

\subsection{Formación biomédica: análisis descriptivo.}

El análisis de medias reportó, de acuerdo con el valor atípico superior $X+1 \sigma=3.19$ que lo más valorado de la formación biomédica por los docentes y estudiantes fue que incluye la revisión de casos clínicos y que busca que los estudiantes desarrollen el pensamiento

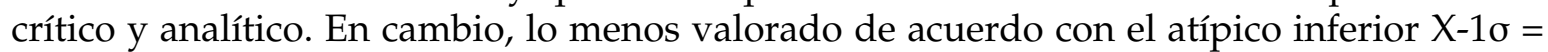
2.83 fue que la facultad forma a los estudiantes para lograr la sostenibilidad ambiental y para prevenir la contaminación (tabla 6).

Tabla 6. Variables Más y Menos valoradas por docentes y estudiantes respecto a la formación biomédica.

\begin{tabular}{|l|c|l|c|}
\hline \multicolumn{1}{|c|}{ Más valorado } & Media/D.E. & \multicolumn{1}{c|}{ Menos valorado } & Media/D.E. \\
\hline $\begin{array}{l}\text { III.45. La formación } \\
\text { biomédica incluye la } \\
\text { revisión de casos clínicos }\end{array}$ & $3.19 \pm 1.05$ & $\begin{array}{l}\text { II.22. La facultad forma } \\
\text { estudiantes para lograr } \\
\text { la sostenibilidad } \\
\text { ambiental y prevenir la } \\
\text { contaminación }\end{array}$ & $2.53 \pm 1.20$ \\
\hline $\begin{array}{l}\text { III.46. La formación } \\
\text { biomédica busca que los } \\
\text { estudiantes desarrollen el } \\
\text { pensamiento crítico y } \\
\text { analítico }\end{array}$ & $3.23 \pm 1.00$ & & \\
\hline
\end{tabular}




\subsection{Diferencias significativas entre estudiantes y docentes respecto al ambiente académico}

En este apartado se muestran los resultados de un estudio comparativo que permite constatar la hipótesis de investigación $\mathrm{H}_{1}$ : existen diferencias significativas entre los estudiantes y docentes de ciencias básicas y clínicas respecto a los elementos que integran el medio ambiente académico. Estas diferencias significativas entre docentes y estudiantes, se observaron en las siguientes variables:

- El ambiente académico contribuye a aprender a aprender; las diferencias se encontraron entre los estudiantes de clínicas y lo estudiantes de básicas siendo los primeros quienes lo consideran más.

- La carga horaria permite a los estudiantes estudiar en la biblioteca y en casa; asimismo que los docentes propician habilidades para la comunicación. Son los docentes de básica y clínica y estudiantes de básicas quienes lo consideran más en comparación con los estudiantes de clínicas.

- Los docentes brindan asesoría académica. Son los docentes de básicas quienes lo consideran más en comparación con los docentes de clínicas.

- La facultad cuenta con servicios de seguridad; se observó que son los docentes de básicas quienes lo valoraron más, en comparación con los estudiantes de básicas y clínicas.

- Los docentes se comunican con los estudiantes de forma asertiva; se observó que son los docentes de básicas y clínicas quienes lo consideran más en relación a los estudiantes de básicas y clínicas.

- Los docentes demuestran una actitud ética. Son los docentes de ciencias básicas y clínicas quienes lo consideran más en comparación con los estudiantes de básicas y clínicas. Asimismo, se observaron diferencias significativas entre los estudiantes siendo los de clínicas, los que los consideran más.

- Los docentes los motivan a aprender a aprender. Son los docentes de básicas y clínicas quienes lo consideran más en comparación con los estudiantes de básicas.

- Los docentes propician el aprendizaje auto dirigido y autorregulado, así como el trabajo colaborativo. Son los docentes de básica quienes lo consideran más que los estudiantes de básicas.

- Los docentes propician el aprendizaje complejo y el pensamiento crítico. Son los docentes de clínicas quienes lo consideran más que los estudiantes de clínicas.

- Los docentes demuestran experiencia profesional, son los docentes de básicas quienes lo consideran más en comparación con los estudiantes de cínicas.

- Los servicios administrativos son eficientes, son los docentes de básicas quienes lo consideran más que los estudiantes de básicas y clínicas.

Con ello se aprecia que los docentes de básicas valoraron en mayor medida, las variables relacionadas con el medio ambiente académico en comparación con los estudiantes de básicas y clínicas.

\subsection{Diferencias significativas entre estudiantes y docentes respecto a la formación biomédica.}

Se muestran los resultados comparativos que permiten constatar la hipótesis de investigación $\mathrm{H}_{1}$ de que existen diferencias significativas entre los estudiantes y docentes de ciencias básicas y clínicas respecto a los elementos que integran el medio ambiente y la formación biomédica. Las diferencias significativas entre docentes y estudiantes se observaron en las siguientes variables de la formación biomédica:

- La facultad promueve el aprendizaje en la interacción con los pacientes; se observó que son los docentes de básicas quienes lo consideran más en comparación con los estudiantes de básicas y clínicas. 
- La facultad forma estudiantes para ser emprendedores, para planear y ejecutar proyectos que beneficien a la sociedad. Son los docentes de básicas y clínicas quienes lo consideran más en comparación con los estudiantes de básicas y clínicas.

- La facultad forma a los estudiantes en valores y ética. Son los docentes de básicas quienes lo consideran más en comparación con los estudiantes de clínicas.

- La facultad forma a los estudiantes para lograr la sustentabilidad ambiental y prevenir la contaminación, para tener una vida saludable; asimismo, los prepara en la utilización de las TICs. En estas variables son los docentes de clínicas quienes lo consideran más en comparación con los estudiantes de clínicas.

Aquí se aprecia que son los docentes de clínicas quienes valoraron más la formación biomédica que los docentes de básicas y los estudiantes de básicas y clínicas.

3.5 Aspectos del medio ambiente académico que se relacionan de forma significativa con la formación biomédica.

En este apartado, se muestran los resultados del análisis de correlación entre las variables simples del medio ambiente y las variables simples de la formación biomédica. Un ambiente académico que garantiza la formación integral como médico se correlaciona significativamente con contribuir a que el estudiante aprenda a aprender y realice investigación médica. Asimismo, con que la facultad promueva la formación del médico humanista, busque que los estudiantes posean el conocimiento necesario para el manejo integral del paciente, la formación biomédica incluya la revisión de casos clínicos y busque que desarrollen el pensamiento crítico y analítico. Asimismo, un ambiente académico que forme estudiantes en valores y ética, así como para trabajar de manera colaborativa se correlaciona de forma significativa con: formar médicos humanistas y estudiantes que posean el conocimiento necesario para el manejo integral del paciente Igualmente, se relaciona significativamente con la revisión de casos clínicos, el desarrollo del pensamiento crítico y analítico, con una formación biomédica dirigida, supervisada, evaluada y realimentada por los docentes y con una formación que comprenda áreas fundamentadas en el conocimiento y razonamiento científico. También resaltó que un ambiente académico que forma a los estudiantes en la investigación médica, se relaciona de forma significativa con: una educación de calidad, con equidad e inclusión, con la formación para ser emprendedores y ejecutar proyectos que beneficien a la sociedad, con formarlos para emplear las tecnologías de la información y la comunicación, con una formación dirigida, supervisada, evaluada y realimentada por los docentes; así como también, con la inclusión de áreas esenciales fundamentadas en el conocimiento y razonamiento científico.

Los docentes que propician habilidades de comunicación, habilidades para un desarrollo profesional continuo y habilidades para el ejercicio profesional futuro, se correlacionan significativamente con: la formación biomédica al propiciar que los estudiantes posean el conocimiento necesario para el manejo integral del paciente, con incluir la revisión de casos clínicos y buscar que los estudiantes desarrollen el pensamiento crítico y analítico. De igual forma, que los docentes se comuniquen de forma asertiva, motiven a sus estudiantes a aprender a aprender, propicien el estudio auto dirigido y/o autorregulado, así como el pensamiento complejo $\mathrm{y}$, apliquen estrategias para el desarrollo del pensamiento crítico, motivando el trabajo colaborativo, se correlaciona significativamente con: formar a los estudiantes para el manejo integral del paciente, que la formación incluya la revisión de casos clínicos, la formación biomédica busque que los estudiantes desarrollen el pensamiento crítico y analítico y sea dirigida, supervisada, evaluada y realimentada por los docentes, asimismo, como comprenda áreas 
fundamentadas en el conocimiento y razonamiento científico. Destacó una relación significativa entre las aulas y los recursos de informática adecuados para el desarrollo de las clases y el aprendizaje con: que la formación biomédica favorezca el manejo integral del paciente, incluya la revisión de casos clínicos y colabore al desarrollo del pensamiento crítico y analítico.

Finalmente, se observó una relación significativa entre los servicios académicos y administrativos que atienden las necesidades de los estudiantes y docentes de manera eficiente con: que la formación biomédica favorezca el manejo integral del paciente, la revisión de casos clínicos, y el desarrollo del pensamiento crítico y analítico. Además, de que la formación sea dirigida, supervisada, evaluada y realimentada por los docentes y comprenda áreas fundamentadas en el conocimiento y razonamiento científico. Este análisis muestra la importancia de la intervención docente en el medio ambiente para favorecer una formación biomédica humanista supervisada y evaluada donde los médicos desarrollan el pensamiento crítico, creativo y complejo para el manejo integral del paciente.

\section{Discusión}

Los resultados mostraron que un ambiente académico que garantiza la formación integral del médico, forme a los estudiantes en valores y ética y propicie en ellos el trabajo colaborativo, se correlaciona significativamente con la formación humanista del médico para el manejo integral del paciente. Esto es relevante ya que el objetivo fundamental de la formación biomédica debe estar orientado a la formación de profesionales preparados para contribuir con los propósitos fundamentales de la medicina, incluyendo el mantenimiento de la salud, el alivio del sufrimiento, la curación de la enfermedad, el control de la pérdida innecesaria de vidas y el acompañamiento durante el tránsito hacia una muerte digna (11). Es uno de los retos que la pedagogía actual analiza con más interés y esperanza en este nuevo milenio, el educar en valores (12). Es así que en el estudio Román y cols., destacaron a la bioética como la herramienta curricular donde los valores y la ética juegan un papel predominante en los procesos formativos de los estudiantes de medicina en busca de una Salud Pública de elevada calidad (13).

En nuestro trabajo hemos visto que entre los aspectos mejor valorados está que el docente motive a sus estudiantes a aprender a aprender y a desarrollar un estudio auto dirigido y/o autorregulado, siendo los docentes de básicas quienes lo consideran más. Favorecer este tipo de aprendizajes en los estudiantes es importante, de acuerdo con un estudio realizado con el fin de medir la percepción del estudiante de grado de medicina, acerca del ambiente educativo en el IMSS, que demostró que los estudiantes que aprenden a su propio ritmo sin fomentar la competencia académica entre ellos, perfeccionan progresivamente sus aptitudes y refinan su quehacer educativo dentro de la institución (9).

Este estudio también muestra que, por parte del docente, el favorecer las habilidades de comunicación, para el desarrollo profesional continuo y para el ejercicio profesional futuro a través de historias clínicas y bitácoras de visitas con pacientes incide en el desarrollo del pensamiento crítico, analítico y complejo para el manejo integral del paciente; esto coincide con un estudio realizado por López y cols. en 2016, cuyo objetivo fue indagar sobre las necesidades percibidas por estudiantes y docentes para desarrollar el pensamiento crítico-analítico, el cual mostró que tanto docentes como estudiantes expresaron la necesidad de implementar estrategias que contribuyan a desarrollar este pensamiento, debido a la importancia que tiene dentro de la formación académica y el desempeño de los futuros profesionales de la salud (14). Otro aspecto que favorece su 
formación biomédica y profesional futura es la investigación; no obstante, fue uno de los aspectos menos valorados en esta investigación.

Otra área de oportunidad identificada es la carga horaria, que no propicia que estudien en casa y en la biblioteca, lo cual además no favorece una vida saludable con ejercicio y alimentación balanceada. Esto coincide con un estudio realizado en una Facultad en Perú, donde se evaluó la calidad del ambiente académico a través de la escala DREEM; en esta investigación, los estudiantes evaluaron negativamente aspectos tales como horario mal programado con mala aplicación del tiempo para la enseñanza y excesivo cansancio (2). Esto cobra relevancia de acuerdo con un estudio, donde se evaluaron los factores asociados al estrés académico en estudiantes de medicina y se encontró que la principal preocupación de los estudiantes está relacionada con la sobrecarga del trabajo, exigencia y desempeño; en este análisis, los estudiantes refirieron dificultades en la gestión del tiempo, particularmente en lo que concierne al equilibrio entre las actividades sociales, las tareas académicas y limitación de las oportunidades para el desarrollo de relaciones personales, lo cual ocasiona en los estudiantes falta de concentración, fatiga intelectual, inapetencia a las tareas habituales, descuido, tristeza, descenso del rendimiento intelectual o físico, nerviosismo, modificación de la frecuencia cardíaca, tensión arterial, metabolismo y actividad física (15); además de afectar negativamente a la motivación del estudiante.

Finalmente, cabe mencionar que una de las limitaciones de este estudio fue la disponibilidad de los participantes para contestar los cuestionarios, ya que debido a que se aplicó de forma virtual y a voluntad de los participantes, se requirió de más tiempo del previsto para recolectar la muestra.

\section{Conclusiones}

- Lo más valorado por docentes y estudiantes respecto al ambiente académico fue que los docentes demuestren una actitud ética, propicien el estudio autodirigido y autorregulado, propicien el pensamiento complejo y el pensamiento crítico, demuestren experiencia en la materia que imparten y que los servicios administrativos sean eficientes.

- Lo menos valorado por estudiantes y docentes respecto al ambiente académico fue que el ambiente académico contribuya a realizar investigación médica, que la facultad forme a los estudiantes para tener una vida saludable, con ejercicio y alimentación acorde con el plato del bien comer, que la carga horaria permita que los alumnos estudien en casa y en la biblioteca y que los estudiantes y docentes conozcan si la facultad cuenta con servicios de seguridad.

- Lo más valorado de la formación biomédica por los docentes y estudiantes fue que incluye la revisión de casos clínicos y que busca que los estudiantes desarrollen el pensamiento crítico y analítico.

- Se encontraron diferencias significativas entre estudiantes y docentes de ciencias básicas y clínicas respecto al medio ambiente académico y la formación biomédica.

- Entre los aspectos del medio ambiente académico relacionados de forma significativa con la formación biomédica se encuentran un ambiente académico que garantice la formación integral como médico, un ambiente académico que forme estudiantes en valores y ética, así como para trabajar de manera colaborativa y una facultad que forma estudiantes en investigación.

- En cuanto al desempeño docente destacó que existe una relación significativa de los siguientes aspectos con la formación biomédica: los docentes propician habilidades de comunicación y habilidades para el desarrollo profesional continuo, propician habilidades para el ejercicio profesional futuro a través de historias 
clínicas y bitácoras de visitas con pacientes y brindan asesoría académica cuando lo solicitan los estudiantes, así como, los docentes se comunican de forma asertiva, motivan a sus estudiantes a aprender a aprender, propician el estudio autodirigido y/o autorregulado, propician el pensamiento complejo, aplican estrategias para propiciar el desarrollo del pensamiento crítico y propician el trabajo colaborativo.

Material suplementario: se proporciona el cuestionario completo.

Financiación: No ha habido financiación.

Agradecimientos: Al comité de editorial de la Revista Española de Educación Médica

Declaración de conflicto de interés: Los autores declaran no tener ningún conflicto de intereses.

\section{Referencias}

1. Hernández AT. Sobre el concepto de formación [Internet]. Revista Milenio 2013 Dic. Disponible en: https://www.milenio.com/opinion/alfonso-torres-hernandez/apuntes-pedagogicos/sobre-elconcepto-de-formacion (visitado el 16/09/2020)

2. Flores-Flores O, Lajo-Aurazo Y, Zevallos-Morales A et al. Ambiente educativo en una facultad de medicina peruana: una tendencia negativa a lo largo de los años académicos. Rev Med Chile. 2018; 146:53-63. Disponible en https://scielo.conicyt.cl/pdf/rmc/v146n1/0034-9887-rmc-146-01-0053.pdf (visitado el 17/08/2020)

3. Torres LV, Blanco A. Ambiente educativo en la escuela de medicina de una Universidad pública: diagnóstico basal. Revista Salud Uis. 2018; 50(4):344-51. Disponible en: https: / / dialnet.unirioja.es/servlet/articulo?Codigo $=6790418$

http://dx.doi.org/10.18273/revsal.v50n4-2018007

4. Olave G, Pérez C, Fasce E, et al. Factores que afectan al ambiente educativo en la formación preclínica de medicina según sus docentes. Rev. Méd. Chile [Internet]. 2016 Oct; 144(10): 1343-1350. https://scielo.conicyt.cl/pdf/rmc/v144n10/art15.pdf. $\quad$ http://dx.doi.org/10.4067/S003498872016001000015

5. García-Gascón A, Querts O, Martínez F, Quesada S. Estrategia pedagógica para la formación profesional en ciencias biomédicas. MEDISAN. 2015; 19(02):266. Disponible en: https://www.medigraphic.com/pdfs/medisan/mds-2015/mds152q.pdf (visitado el 08/01/21)

6. Parra-Acosta H, Benavides-Olivera J, López-González JC, et al. Evaluación por competencias de un modelo novedoso de gestión de calidad en médicos internos de pregrado. Revista investigación en educación medica. 2014; 3(10):65-73. Disponible en: http://riem.facmed.unam.mx/node/242

7. https://doi.org/10.1016/S2007-5057(14)72729-2

8. Cevallos JS. Competencias del docente de la carrera de medicina. Práctica Familiar Rural. 2019; 4(3):135-8. Disponible en: https://practicafamiliarrural.org/index.php/pfr/article/view/134/144

9. https://doi.org/10.23936/pfr.v4i3.134

10. Vera-Carrasco O. El aprendizaje basado en problemas y la medicina basada en evidencias en la formación médica. Rev. Méd. La Paz. 2016; 22(2): 78-86. Disponible en: http://www.scielo.org.bo/scielo.php?script=sci arttext\&pid=S1726-89582016000200013 (visitado el $08 / 01 / 21)$

11. Morales-Gómez A, Medina-Figueroa AM. Percepción del alumno de pregrado de medicina, acerca del ambiente educativo en el IMSS. Revista Médica del Instituto Mexicano del Seguro Social. 2007; 45(2):123-31. Disponible en: https://www.medigraphic.com/pdfs/imss/im-2007/im072e.pdf (visitado el 07/11/2020)

12. Hernández R, Fernández C, Baptista P. Metodología de la investigación. McGraw-Hill Educación; 2014. Disponible en: https://www.uca.ac.cr/wp-content/uploads/2017/10/Investigacion.pdf

13. (visitado el $08 / 01 / 21$ )

14. Morales-Ruiz J.C. Formación integral y profesionalismo médico: una propuesta de trabajo en el aula. Educ. Méd. 2009; 12(2): 73-82. Disponible en: http://scielo.isciii.es/scielo.php? script=sci_arttext\&pid=S1575-18132009000300003 (visitado el 17/12/20) 
15. Selva A, Álvarez J, Calderín I, et al. Educación en valores para la formación integral de estudiantes de medicina desde un enfoque psicopedagógico. MEDISAN 2012; 16(1): 62-66. Disponible en: http:/ / scielo.sld.cu/scielo.php?script=sci arttext\&pid=S1029-30192012000100009 (Visitado el 17/08/2020)

16. omán CA, Ortiz F, Hernández Y. La bioética y la educación en valores en estudiantes de Medicina durante la etapa preclínica: Un análisis desde el enfoque histórico cultural. Revpanorama. 2008; 3(3):22-8. Disponible en: http://revpanorama.sld.cu/index.php/panorama/article/view/146/pdf (visitado el 16/12/2020)

17. López-Silva B, García-Rodríguez I, Hernández MI, et al. El pensamiento crítico-analítico en estudiantes del área de Biología de la Universidad de Guayaquil, Ecuador. EDUMECENTRO 2016; 8(3): 38-51. Disponible en: http://scielo.sld.cu/scielo.php?script=sci_arttext\&pid=S207728742016000300004\&lng=es (Visitado el 23/09/2020)

18. Maceo-Palacio O, Maceo-Palacio A, Ortega Y, et al. Estrés académico: causas y consecuencias. Multimed.

2013;

17

(2):

185-96.

Disponible

en:

http:/ / www.revmultimed.sld.cu/index.php/mtm/article/view/302/411 (visitado el 19/10/2020)

(c) 2021 por los autores. Enviado para su publicación en acceso abierto bajo los términos

$\mathrm{y}$ condiciones de la licencia Creative Commons Attribution (CC BY)

(http://creativecommons.org/licenses/by/4.0/). 